\title{
IoT Automation for Emergency Environments
}

\author{
Mrs.M.Buvaneswari M.E, S.Punitha, Ms.P.Sneka, Ms.G.Sujithra \\ ${ }^{l}$ (Ass.Prof CSE) VSB College of Engineering Technical Campus \\ ${ }^{2}$ B.E. Computer Science and Engineering VSB College of Engineering Technical Campus
}

\begin{abstract}
An emerging technology, IoT enables us machine to machine communication. For communication in emergency environment, IoT is considered as a strong technology. An emergency alert service offered by Terrestrial Digital Multimedia Broadcasting (T-DMB) is known as Automatic Emergency Alert Service (AEAS). This paper illustrates a consumer transceiver for long range communications of IoT in emergency environments. In order to overcome the drawbacks of AEAS, signaling method is used in proposed system. The IEEE802.11ah is a Wi-Fi protocol as transceiver which is under standardization for low cost and low power services.
\end{abstract}

Keywords: TDMB, AEAS, IEEE802.11ah, IR sensor, Gas sensor, Xampp, MP Lab.

\section{Introduction}

In severe emergency situations an effective alert delivery is required for saving human lives. A various communication systems are utilized for rapid, effective and efficient alert delivery. Especially, for the delivery of emergency alert using broadcasting system since they are robust to the destruction of network infrastructure. For a flexible broadcasting system in addition, mobility has been added to digital broadcasting system. In AEAS standard, T-DMB control channel is included as the alert service channel. A T-DMB control information will limit the usage of AEAS. When the usage AEAS is extremely restricted while the T-DMB channel is heavily occupied by T-DMB control data. If the receiver is disabled in mobile station

(MS), then the T-DMB receiver does not have any functionality to receive an AEAS message. Moreover, in every system a wakeup method is required for the disabled T-DMB receiver in emergency situations but, in this system additionally AEAS messages are delivered to all MS users.

This paper depicts, Signaling Emergency Alert System (SEAS) is proposed, the conventional AEAS shortcomings are compensated. Even in the case of conventional T-DMB channel is heavily congested the SEAS allows effective and rapid delivery of emergency alert messages. The receiver performance is enhanced due to the periodic feature allows more sophisticated channel estimation method. To avoid the involvement of the channel repeaters used in T-DMB systems, the SEAS is implemented in the repeater. The users in the service area of the repeater, such implementation inherently offers efficient LBS. This made the system to avoid consumption for LBS in mobile system.

\section{Configuration of T-DMB Emergency Broadcasting System}

The T-DMB EBS can broadcast many emergency alert contents to the environment without any additional operation of the receiver. In general environment, the system can provide T-DMB repeating service for the developed system is composed on-channel repeater part and EBS part. The radio frequency (RF) signal is converted onto intermediate frequency (IF) signal is done after filtering and low noise amplifier (LMA). The base band signal is converted to IF signal by IF up converter and digital to analog converter is implemented after interference cancellation and equalization. A general repeated T-DMB signal and emergency broadcasting signal is function as selected transmission signal executed by 'Switch' block. The switch selects the general IF T-DMB signal and transmits the signal to RF up converter in common environment. 


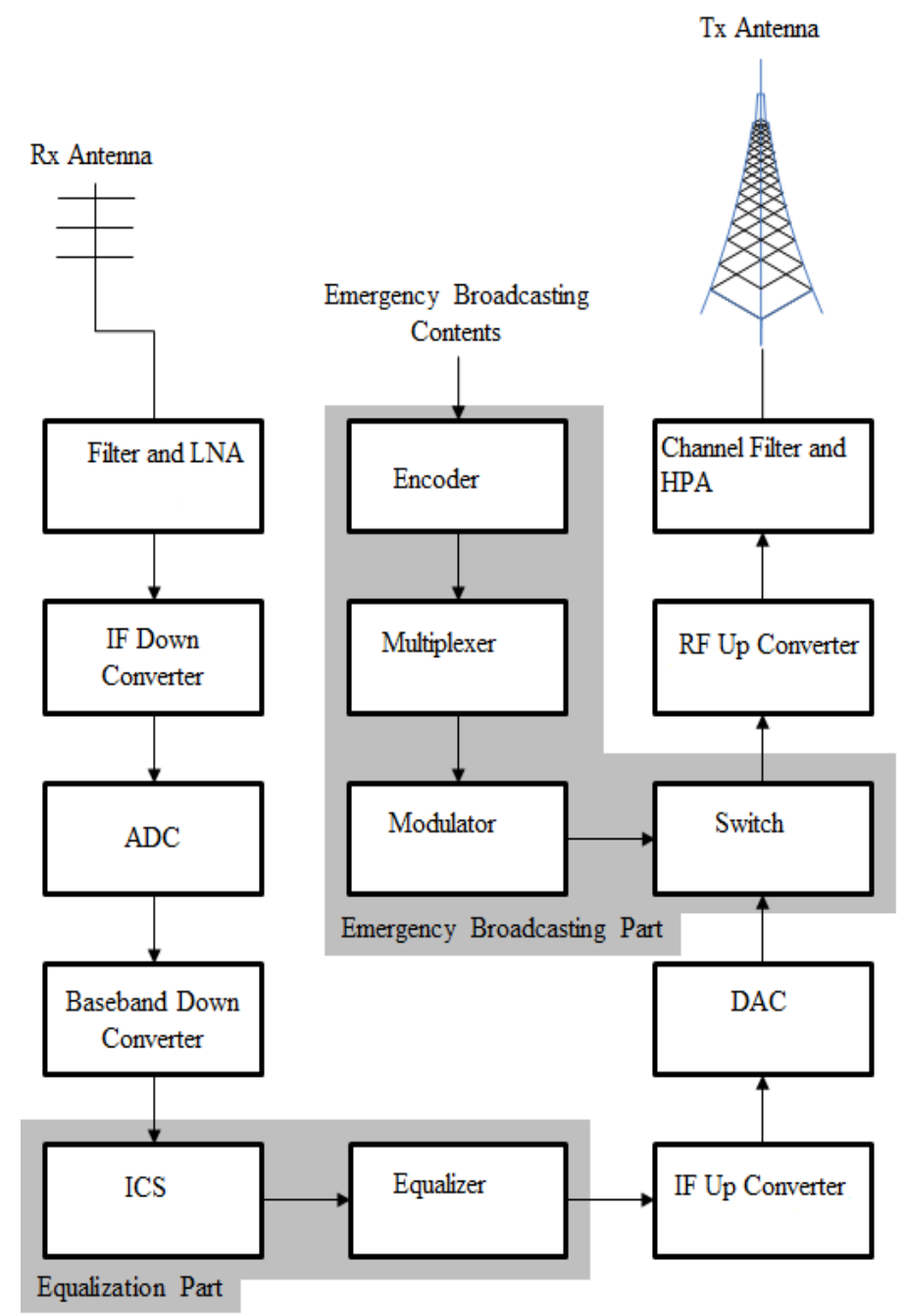

The contents of emergency broadcasting system are encoded in accordance with broadcasting service. The developed T-DMB EBS is providing emergency alert broadcasting to legacy to receivers without any receiver operation additionally. It is very important to change the general broadcasting contents to emergency alert broadcasting contents. According to all eleven services the encoder has to encode the input emergency broadcast content. The encoder eleven contents are multiplexed into three ensembles for transmission. This multiplexing function executed by 'Multiplexer'.

The T-DMB EBS transmits the emergency broadcasting content. The T-DMB on-channel repeater function and EBS function was executed by the system and three ensembles and eleven contents. Demodulator, channel estimator, inverse convertor and adaptive filter are the equalization parts. By using inverse convertor the estimated channel is converted into time domain signal. The multipath signal was removed by equalization part efficiently.

\section{IEEE802.11AH Protocol}

The IoT related service include smart grid scenario based on IEEE802.11 AH and backhaul network. In order to support significant improvements in the efficiency, reliability and sustainability of services devised by smart grid system. The wireless smart metering utility network (Wi-SUN) belongs to WPAN system. To manage and control the home utility data is attached to the home by Wi-SUN via the IEEE802.15.4g protocol. To support short range communication, the IEEE802.15.4g is a kind of WPAN is used. The difference between the smart meter and WPAN are $15.4 \mathrm{~g} / 11 \mathrm{ah}$ GW is relatively short. The IEEE802.11ah AP is converted data by GW retransmits via IEEE802.11ah Wi-Fi protocol. The distance between the GW home smart meters is comparatively shorter than the distance between AP and GW. 


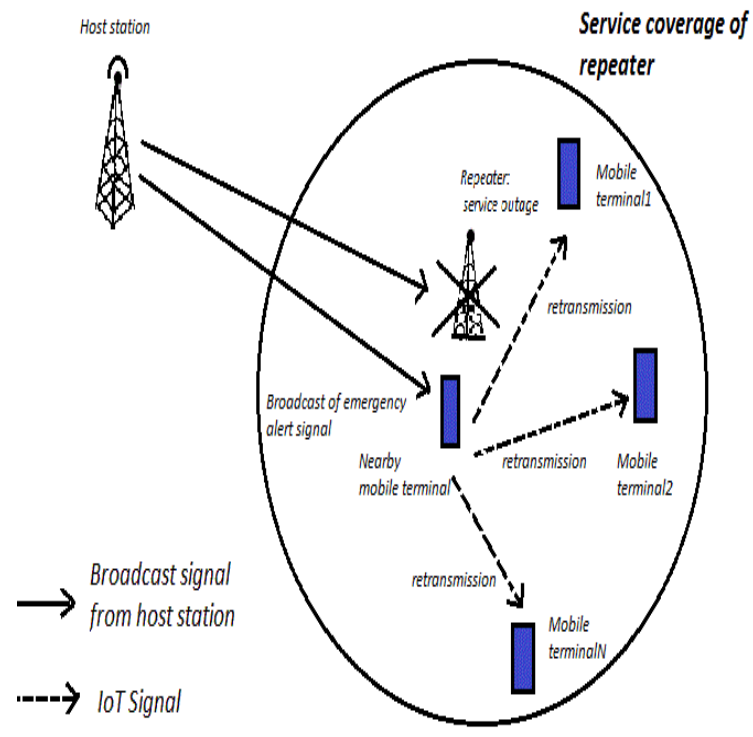

Based on the utility data, a decision for optimal usage of utility data is determined by control and database, and transmits the home smart meter from decision command via the backhaul network and IEEE802.11ah protocol. The conventional architecture of transceiver for IEEE802.1ah under modulation and coding scheme1, BCC, and $2 \mathrm{MHz}$ bandwidth. The transmitter of the system consists of scrambler BCC interleave among RF. The data format in OFDM symbol for Wi-Fi protocol at $2 \mathrm{MHz}$ bandwidth. The null data exist at the remaining 12 subcarriers are indicated; only 52 subcarriers are utilize for a data transmission in one OFDM symbol. The service coverage of IoT should be equivalent to that of repeater. In order to fully support the mobile terminals with the service range of repeater.

\section{Block Diagram}

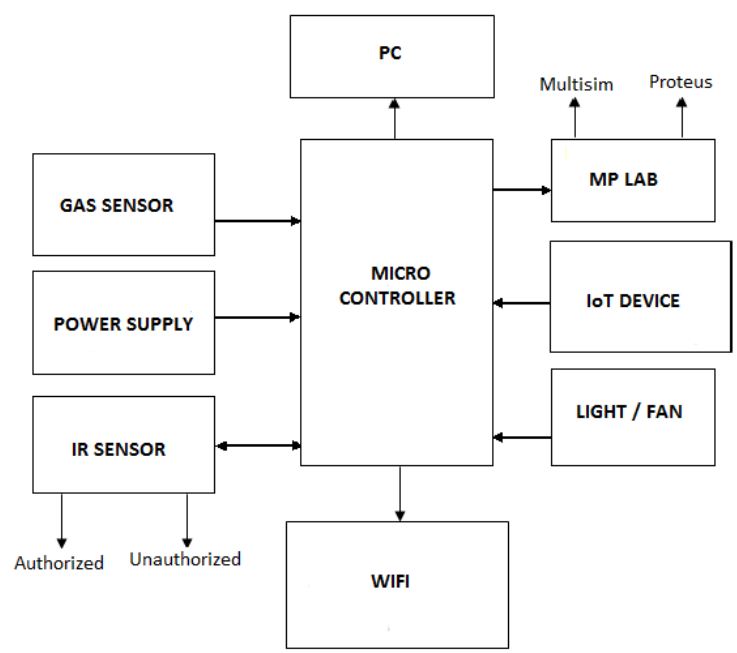

\section{Proposed System}

To overcome the disadvantage of existing system, in this system sensors are used. In this system there are three modules; First module is of designing a web page using Dream viewer and Xampp software. A Xampp (Cross platform (X), Apache (A), My SQL (M), Php (P) and PERL (P) .The developer to create a local web server for testing purposes and it is simple, lightweight Apache distribution. It is a free and open source cross platform web server. It consisting Apache Http sever and interpreters for scripts written in Php and PERL programming languages. It is also called as Wamp. Dream viewer is an application used by web designers and web developers to create websites and applications. Using the web page only the user can access the system. This part is mainly used to Switch ON/OFF the light, fan and leakage of gas. Second module will be the hardware part, sensors like MQ4 Gas sensor, IR sensor and MP lab proteus software to install. Third module will be the combination of hardware and software by using Php and embedded C. For the long range communication IEEE802.11ah Wi-Fi protocol is used. 


\section{Conclusion}

This paper includes based on security and IR sensor, Gas sensor for emergency environment. This paper depicts long range IoT communications based on IEEE802.11ah Wi-Fi protocol. Here we use Wi-Fi to send the data through the internet. In IoT device all the data has been stored and monitoring has been done through the server. When the device is switched on then the Wi-Fi has been connected automatically through the internet and data has been shared. This project is designed with the hope that it is very much economical and helpful to the security purpose and it provides flexibility in operation.

\section{Reference Paper}

[1]. M. Song, S. Chang, and Y.-T. Lee, “A wake-up consumer device for enhanced emergency alert service in T-DMB systems,” IEEE Trans. Consumer Electron., vol. 61, no. 3, pp. 286-294, Aug. 2015.

[2]. K. Jun and S. Chang, "An interference-aware clustering based on genetic algorithm for cell broadcasting service," IEICE Trans. Fund., vol. e96-a,no. 12, pp. 2740-2744, Dec. 2013.

[3]. Y.-H. Lee, G. Kim, S.-R. Park, Y.-T. Lee, and N. Kim, "An efficient emergency broadcasting signal multiplexing method for supporting the legacy T-DMB receivers in break-in system," IEEE Trans. Consumer Electron., vol. 57, no. 4, pp. 1550-1555, Nov. 2011.

[4]. S. Tarkoma and H. Ailisto, "The internet of things program: the Finnish perspective," IEEE Commun. Mag., vol. 51, no. 3, pp. 1011, Mar. 2013

[5]. A. Zanella, N, Bui, A. Castellani, L. Vangelista, and M. Zorzi, "Internet of things for smart cities," IEEE Internet of Things Journal, vol. 1, no. 1, pp. 22-32, Feb. 2014.

[6]. L. Catarinucci, D. de Donno, L. Mainetti, L. Palano, L. Patrono, M. L. Stefanizzi, and L. Tarricone, "An IoT-aware architecture for smart healthcare systems," IEEE Internet of Things Journal, vol. 2, no. 6, pp. 515-526, Dec. 2015.

[7]. I. Jeon, M. Song, S. Chang, S. J. Choi, and Y.-T. Lee, "A signaling emergency alert system multiplexed with T-DMB channel for emergency alert service,” IEEE Trans. Consumer Electron., vol. 61, no. 1, pp. 16-23, Feb. 2015.

[8]. R. Porat, S. K. Yong, and K. Doppler, IEEE P802.11 Wireless LANs: TGah Channel model.

[9]. M. Song, K. Jun, and S. Chang, "An efficient multiplexing method of TDMB and cell broadcast service in emergency alert systems," IEEE Trans. Consumer Electron., vol. 60, no. 4, pp. 549-557, Nov. 2014.

[10]. S.-J. Choi, D.-B. Kwon, J.-Y. Kim, K.-S. Oh, T.-U. Chang, and Y.-K. Hahm, "Design of T-DMB automatic emergency alert service standard: part 1 requirements analysis,” Journal of Broadcast Engineering, vol. 12, pp. 230-241, 2007. 